\title{
ESTUDO DE ANÁLISE TERMOGRAVIMÉTRICA DE DIFERENTES BIOMASSAS LIGNOCELULÓSICAS UTILIZANDO A ANÁLISE POR COMPONENTES PRINCIPAIS
}

\section{STUDY OF THERMO-GRAVIMETRIC ANALYSIS OF DIFFERENT LIGNOCELLULOSIC BIOMASS USING PRINCIPAL COMPONENT ANALYSIS}

Magale Karine Diel Rambo ${ }^{1}$, Michele Cristiane Diel Rambo ${ }^{2}$, Karla Jackeline Costa Rodrigues ${ }^{1}$ Almeida, Gerso Pereira Alexandre ${ }^{1}$

Universidade Federal do Tocantins ${ }^{1}$, Instituto Federal do Tocantins ${ }^{2}$

\begin{abstract}
Resumo
O comportamento térmico de biomassas lignocelulósicas utilizando a termogravimetria e a termogravimetria derivada são importantes, pois relatam a presença de constituintes químicos, tais como hemicelulose, celulose e lignina. E a utilização de uma análise exploratória multivariada, como a análise por componentes principais (PCA) é útil no sentido de agrupar termogramas com perfis semelhantes. Assim biomassas de capim, café, engaço de banana e açaí com comportamentos semelhantes apresentaram maior degradação térmica atribuída à decomposição da hemicelulose, enquanto que para a decomposição da celulose as amostras de arroz, serragem e soja. Observa-se que todas as dez amostras de biomassa exibem tendência semelhante de perda de peso associada à decomposição da lignina.
\end{abstract}

Palavras-chave: Resíduos agro-industriais. Resíduos agro-florestais. Quimiometria. Termogravimetria derivada.

\begin{abstract}
The thermal behavior of lignocellulosic biomasses using thermogravimetric and derivative thermogravimetric are important because they related to the presence of chemical constituents such as hemicellulose, cellulose and lignin. And the use of a multivariate exploratory analysis such as principal component analysis (PCA) is useful in order to group thermograms with similar profiles. Thus biomass such as grass, coffee, banana stalk and açai with similar behaviors exhibited thermal degradation attributed to the decomposition of the hemicellulose, whereas for the cellulose decomposition samples of rice, sawdust and soybeans. It is observed that all the ten biomass samples exhibit similar weight loss trend associated with the decomposition of lignin.
\end{abstract}

Keywords: Agricultural wastes. Forest wastes. Chemometrics. Derivative thermogravimetric. 


\section{Introdução}

Biomassas lignocelulósicas representam uma grande e ainda inexplorada fonte de matéria-prima para processos de conversão térmica (Jeguirim et al., 2014; Chen et al., 2015; Saldarriaga et al., 2015). No Brasil, enormes quantidades de biomassa na forma de resíduos agroindustriais e agro-florestais são produzidos (Rambo et al., 2015), tais como; cascas de café, cascas de coco, cascas de arroz e soja, serragem de eucalipto,bambu, caroço de açaí, capim elefante, caule e engaço de bananeira, entre muitos outros.

Essa biomassa é composta basicamente de três principais componentes que consistem de cerca de $30-50 \%$ de celulose, $15-35 \%$ hemicelulose e $10-20 \%$ de lignina (Limayem e Ricke, 2012). Estudos prévios da análise térmica de materiais lignocelulósicos revelaram que a degradação apresenta a seguinte tendência: umidade, hemicelulose, celulose e por final a degradação da lignina (Raveendran et al.,1996; Yang et al., 2004).

Para que essas biomassas sejam bem aproveitadas é de extrema importância o conhecimento do comportamento térmico dessas durante $\mathrm{o}$ processo de termoconversão. $\mathrm{O}$ conhecimento de tal processo é fundamental para o monitoramento das condições de processamento desses materiais. As técnicas de análise térmica (TGA), particularmente a termogravimetria (TG) e a termogravimetria derivada (DTG) possibilitam a obtenção dessa informação de maneira simples e rápida (Tomczak et al., 2007).

A TGA é um método que determina a mudança de peso das amostras em função da mudança de temperatura. Parâmetros como tempo, peso e temperatura são essenciais. No entanto a TGA sozinha não é suficiente para interpretar a perda de peso da amostra (Parthasarathy et al., 2013). Assim a DTG é utilizada, pois ao aplicarmos as operações de derivação aos termogramas brutos, as informações contidas são geralmente acentuadas (bandas mais estreitas) e se tem uma aparente melhora na resolução do termograma.

A análise por componentes principais (PCA) tem sido muito utilizada, com inúmeras publicações na literatura, auxiliando no trabalho de análise exploratória dos dados, entre outras aplicações (Rambo et al., 2013; Rambo et al., 2015b). Nesse estudo, a PCA foi aplicada nos termogramas derivados para indicar as semelhanças e diferenças entre as diversas biomassas e avaliar as etapas que afetam tais matérias-primas durante o processo de termodegradação.

\section{Material e Métodos}

\subsection{Amostras}

Dez diferentes biomassas (caroço de açaí, casca de café, serragem de eucalipto, casca de arroz, casca de soja, casca de coco, capim elefante, bambu, engaço e pseudocaule de banana) correspondentes a uma fração úmida e não moída foram devidamente coletadas de diversas regiões do Brasil no período de março 
2010 - março 2012. Essas amostras foram mantidas congeladas em sacos plásticos até as etapas seguintes do processo. Após secas (em bandejas a temperatura ambiente) foram moídas (em um moinho tipo faca da marca MA 920- Marconi) e em sequencia classificadas em agitador de peneiras automático (VP-01, Bertel) para análises granulométricas e para redução do tamanho das partículas (180 $\mu \mathrm{m}$ a $850 \mu \mathrm{m})$. Após o processo de redução, as amostras foram secas novamente mas dessa vez com o objetivo de se determinar o teor de umidade (em estufa da marca 315 Se- Fanem a $105 \pm$ $5{ }^{\circ} \mathrm{C}$ por um período de 8 horas)

Ao final do processo, as amostras foram armazenadas em recipientes herméticos e então caracterizadas por processos térmicos.

\subsection{Análise térmica}

A análise térmica foi avaliada por meio das técnicas de análise de TG e DTG. A termogravimetria foi realizada em um equipamento TG 209 Netzsch, na faixa de temperatura de $20{ }^{\circ} \mathrm{C}$ a $800{ }^{\circ} \mathrm{C}$, em atmosfera de nitrogênio, sob fluxo de 15 mL.min ${ }^{-1}$ e taxa de aquecimento de 20 ${ }^{\circ} \mathrm{C}$. $\mathrm{min}^{-1}$. As amostras utilizadas para a análise termogravimétrica foram 4 a $6 \mathrm{mg}$ de biomassa na forma de pó. As perdas de massa associadas com certos intervalos de temperatura são consideradas um indicativo da quantidade de componentes químicos diferentes presentes na biomassa.

\subsection{Análise multivariada dos Dados}

As análises multivariadas foram realizadas utilizando o software Unscrambler 10.3 para PCA (Camo, Noruega) e o Origin 6.0 (Originlab) para construção dos gráficos e figuras ilustrativas (StatSoft, EUA, 2005).
A Associação Americana para Testes e Materiais (ASTM) define a PCA como um procedimento matemático de projeção que projeta a matriz de dados com muitas variáveis, em um espaço de dimensão menor, reduzindo a dimensionalidade do conjunto dos dados. Ou seja, um novo sistema de eixos é construído, que podem ser denominados fatores, componentes principais ou variáveis latentes, para representar a matriz de dados. Como resultado, as informações mais importantes e relevantes se tornam mais fáceis de serem interpretadas. Essas novas componentes (PC) são obtidas em ordem decrescente de variância, de forma que a primeira componente principal (PC1) tem a maior variância possível (ou seja, representa o máximo da variabilidade nos dados). Cada componente seguinte, por sua vez, tem a máxima variância residual, não representada pelas componentes anteriores, sob a restrição de ser ortogonal aos componentes anteriores, dessa forma, as componentes principais são não-correlacionadas. $\mathrm{O}$ número máximo de componentes, que podem ser obtidos com essas condições, é menor do que ou igual ao número de variáveis ou de amostras, o que for menor. Há inúmeras excelentes descrições tratando a base matemática da PCA (Wold et al., 1987; Brereton, 1992).

A PCA foi realizada utilizando-se os termogramas derivados ( $1^{a}$ Derivada) com os dados centrados na média e 4 fatores foram aplicados. A PCA foi utilizada para análise exploratória dos dados verificando possíveis agrupamentos das biomassas, ou seja, a relação entre as amostras (escores) e as variáveis (pesos). 
3 Resultados e Discussões

A Figura 1 mostra as curvas TG e DTG para todas as biomassas em questão.
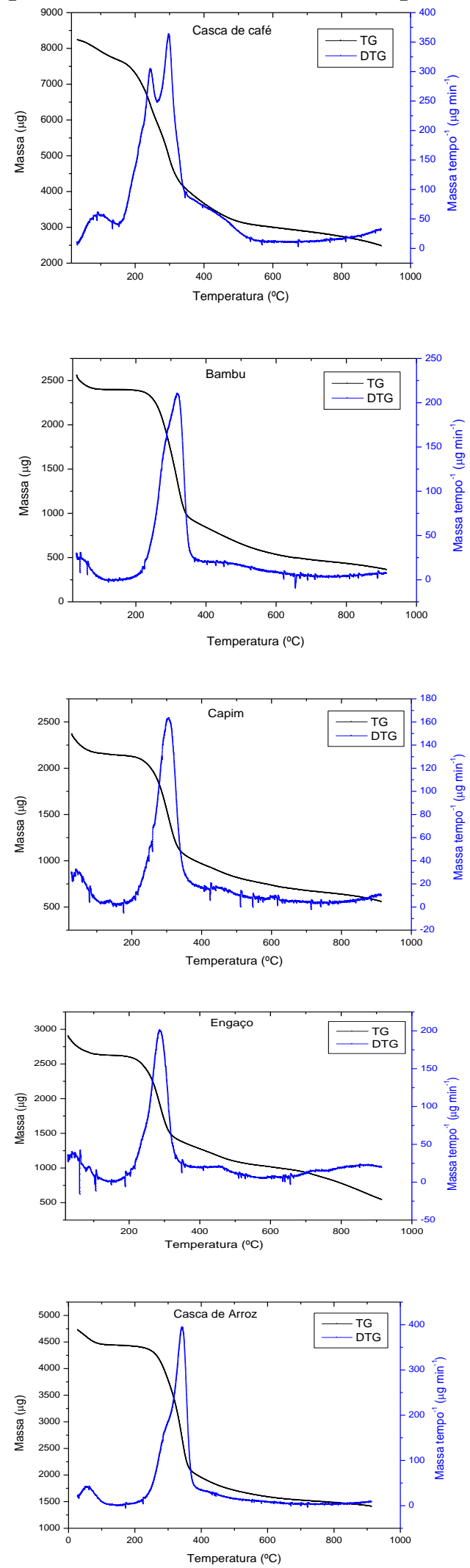
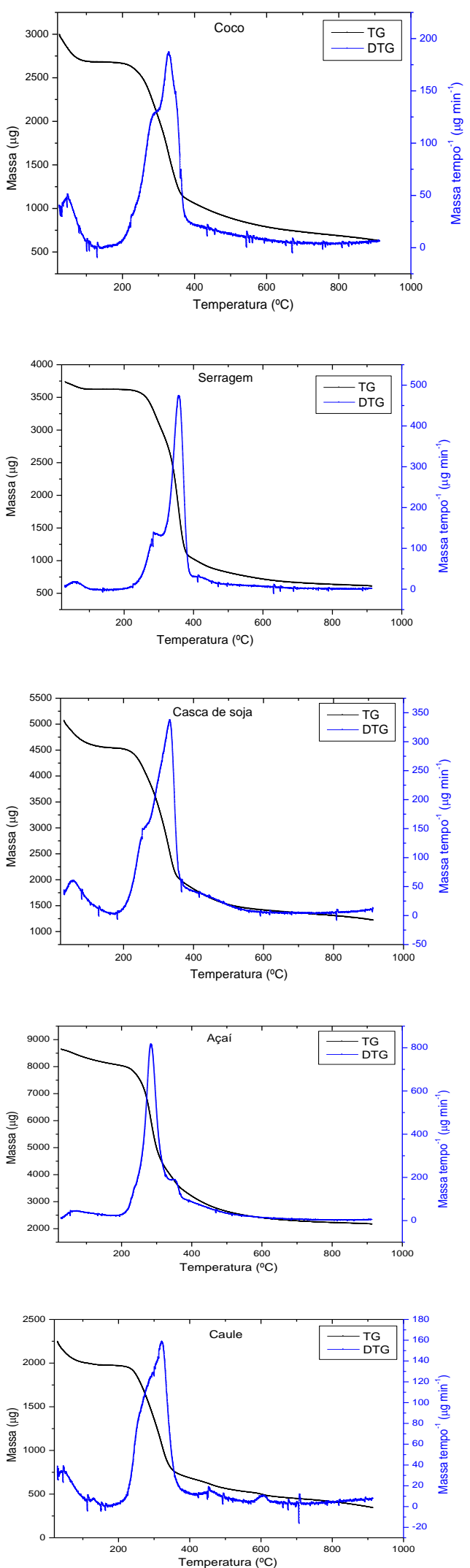

Figura 1. Análises termogravimétricas (TG) e suas derivadas (DTG) das biomassas lignocelulósicas. 
Estas apresentam um comportamento típico de degradação térmica para biomassas lignocelulósicas, com três etapas bem definidas: liberação de umidade na faixa de 50 a $100{ }^{\circ} \mathrm{C}$, decomposição de carboidratos (hemicelulose e celulose) no intervalo de 250 a $400{ }^{\circ} \mathrm{C}$, acima de $400{ }^{\circ} \mathrm{C}$ uma degradação contínua e lenta associada a lignina e ao final do processo ocorre a formação de carvão e cinzas.

Os resultados da PCA dos termogramas derivados são úteis a fim de agrupar amostras com perfis semelhantes (Figura 2) e compreender a relação dessas amostras com as variáveis (Figura 3).
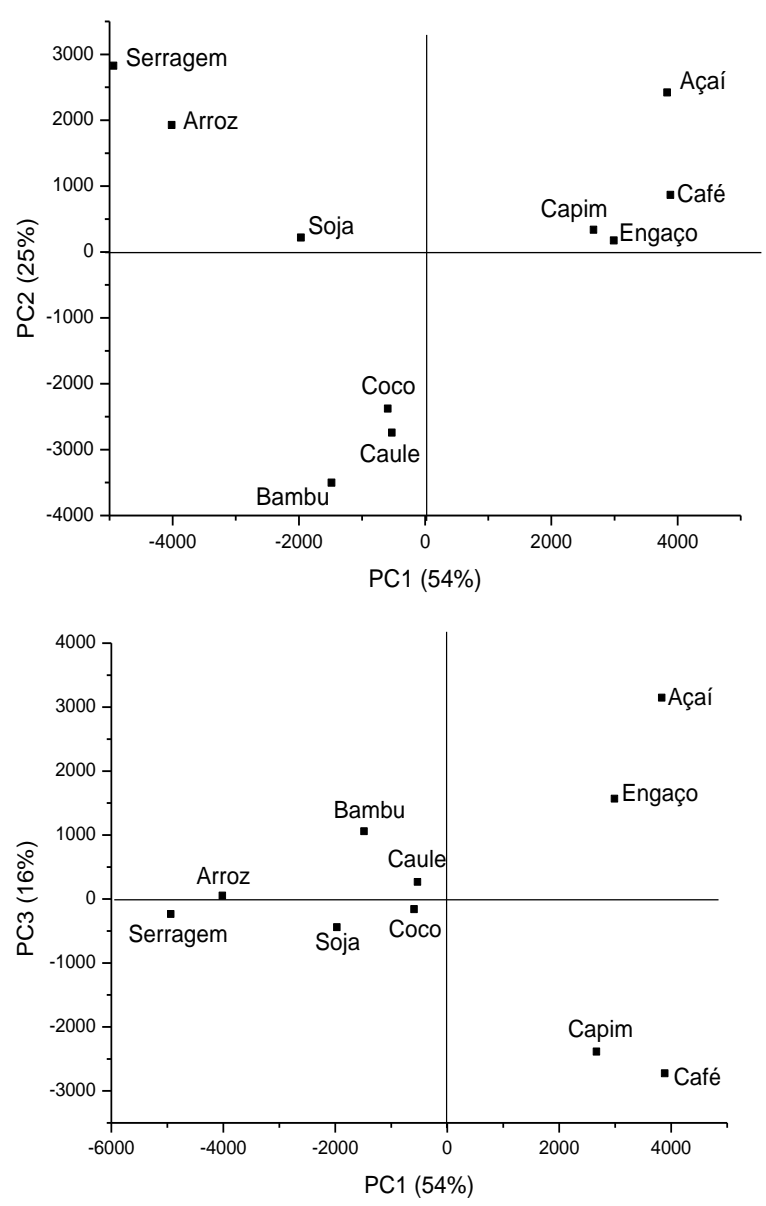

Figura 2. Gráfico de escores das duas primeiras componentes principais (A) e gráfico de escores da primeira e da terceira PC (B).
As duas primeiras PC explicam 54\% e $25 \%$ da variância total, respectivamente. A PC1 apresentou pesos positivos e pesos negativos para as temperaturas de $290{ }^{\circ} \mathrm{C}$ e $340{ }^{\circ} \mathrm{C}$, respectivamente (Figura 3), indicando que as amostras de capim, café, engaço de banana e açaí com escores positivos na PC1, apresentaram maior degradação térmica nessa temperatura (290 ํ), atribuída a decomposição da hemicelulose (Jeguirim et al., 2014). A PCA confere com o perfil das curvas TG e DTG para essas amostras (Figura 1 e Figura 4), com picos mais intensos antes de $300{ }^{\circ} \mathrm{C}$.

A PC1 negativa associada à temperatura de $340{ }^{\circ} \mathrm{C}$ é atribuída à decomposição da celulose, na qual as amostras de arroz, serragem e soja apresentam um perfil no termograma derivado (pico) mais intenso nessa região (Figura 1). O grupo formado por coco, caule e bambu apresenta um comportamento mais ameno (picos menos intensos) quando comparado às amostras dos demais grupos (Figura 4).

Por outro lado, a PC2 foi caracterizada por duas bandas positivas (290 e $340^{\circ} \mathrm{C}$ ), como dito anteriormente, atribuídas a decomposição de hemicelulose e celulose, respectivamente. Os escores da PC2 corroboram a justificativa da PC1, no qual as biomassas com perfis mais intensos (Figura 4) antes e após $300{ }^{\circ} \mathrm{C}$ correspondem a degradação dos polissacarídeos. O grupo formado por coco, caule e bambu (picos amenos) não foram detectados pela PC2.

A PC3 com apenas $16 \%$ da variância explicada, foi responsável pela distinção de amostras associadas à degradação de compostos orgânicos voláteis - extrativos $\left(240^{\circ} \mathrm{C}\right)$, tais como capim, café, coco, soja 
e serragem. Ao observamos as derivadas contínua e lenta, atribuída à degradação de tais amostras (figura 4), geralmente da lignina (Guimarães et al., 2009; apresentam uma pequena elevação Parthasarathy et al., 2013; Chen et al., (ombro) associados à temperatura de 240 2015).

${ }^{\circ} \mathrm{C}$.
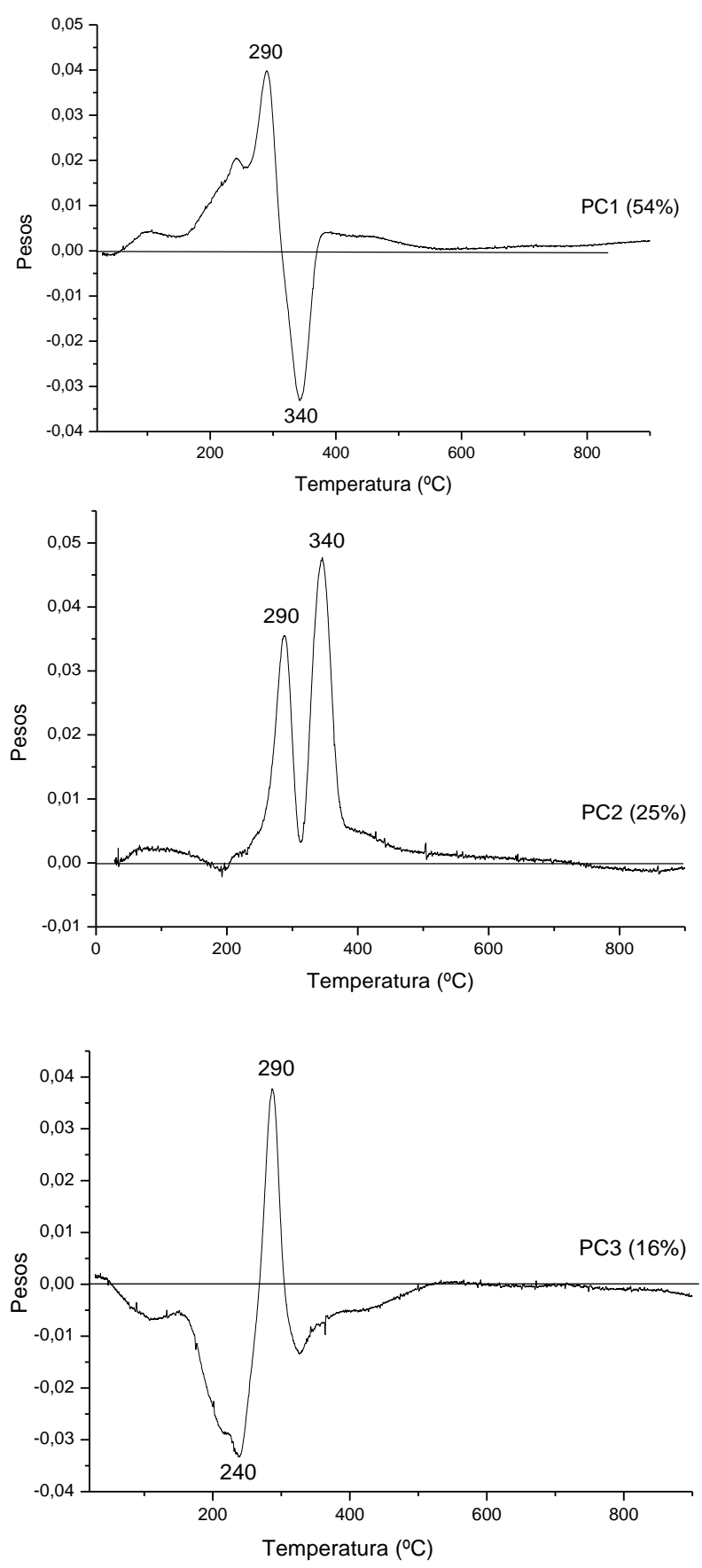

Figura 3. Gráfico de pesos da análise PCA.

Observando os gráficos de pesos (Figura 3) percebe-se que para todas as componentes principais (PCs) acima de $400{ }^{\circ} \mathrm{C}$ tem-se uma perda de peso
A variação entre as temperaturas de degradação entre as diferentes biomassas lignocelulósicas pode ser atribuída ao fato das diferentes composições químicas de cada matéria-prima. No estudo publicado previamente por Rambo et al., (2015a) é possível observar a composição físico-química de inúmeros resíduos lignocelulósicos.

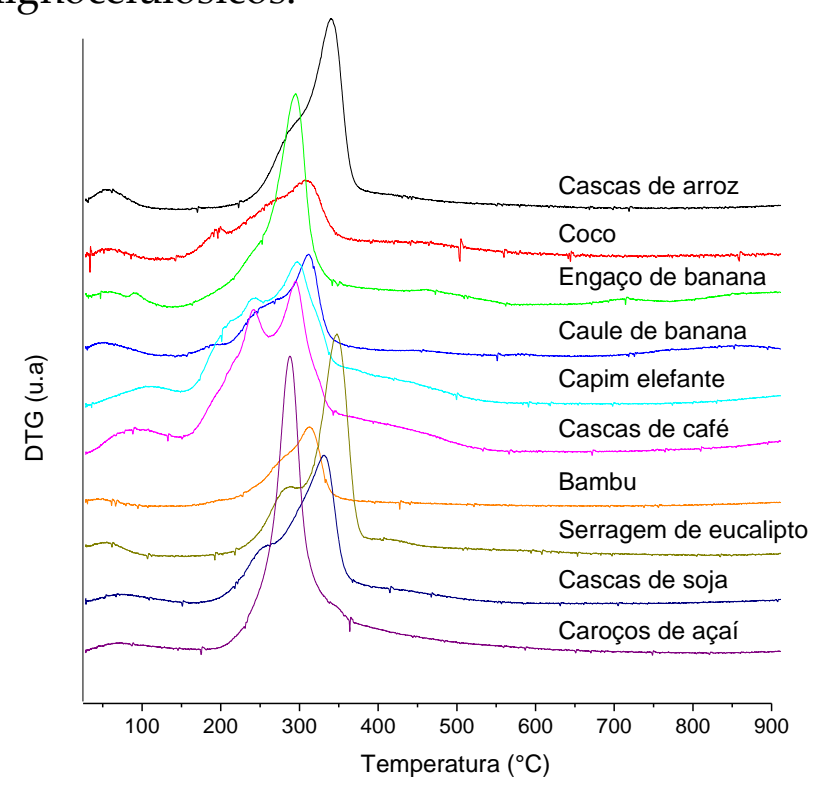

Figura 4. Análises termogravimétricas (DTG) das biomassas sobrepostas.

\section{Conclusões}

A análise térmica das 10 biomassas pode ser dividida em três estágios que correspondem à decomposição da hemicelulose, celulose e lignina. Aplicando derivadas aos termogramas foi possível avaliar de forma mais exata as faixas de degradação de cada composto.

A PCA forneceu resultados úteis no sentido de facilitar a compreensão de biomassas com perfis semelhantes e ainda avaliar as variáveis que mais interferem no processo de degradação térmica de cada biomassa. 


\section{Agradecimentos}

Ao CNPq (420154/2013-2) pelo apoio financeiro.

\section{Referências}

ASTM E 1655-00. Standard Practices for Infrared Multivariate Quantitative Analysis; 2005.

Jeguirim, M.; Bikaia, J.; Elmaya, Y.; Limousya, L.; Njeugna,E.Thermal characterization and pyrolysis kinetics of tropical biomass feedstocks for energy recovery. Energy Sustain. Dev. 2014;23: 188-193.

Parthasarathy, P.; Narayanan, K.S.; Arockiam, L. Study on kinetic parameters of different biomass samples using thermo-gravimetric analysis. Biomass Bioenerg. 2013;58:58-66.

Chen, Z. et al. Characteristics and kinetic study on pyrolysis of five lignocellulosic biomass via thermogravimetric analysis. Bioresour. Technol. 2015; doi: http://dx.doi.org/10.1016/j.biortch.2015.05 .062 .

Saldarriaga, J, F.; Aguado, R.; Pablos, A.; Amutio, M.; Olazar, M.; Bilbao, J. Fast characterization of biomass fuels by thermogravimetric analysis (TGA). Fuel, 2015;140:744-75.

Limayem,

A.; Ricke,

S.C. Lignocellulosic biomass for bioethanol production: Current perspectives, potential issues and future prospects. Prog. Energ. Combust. 2012;38:449-467.

Raveendran, K.; Ganesh A.; Khilar, K.C. Pyrolysis characteristics of biomass and biomass components. Fuel, 1996;75: 987-98.

Yang, H.; Yan, R.; Chin, T.; Liang, D.T.; Chen, H.; Zheng, C. Thermogravimetric analysis-Fourier transform infrared analysis of palm oil waste pyrolysis. Energ. Fuel, 2004;18:1814-1821.

Tomczak, F.; Sydenstricker, T.H.D.; Satyanarayana, K.G. Studies on lignocellulosic fibers of Brazil. Part II: Morphology and properties of Brazilian coconut fibers. Composites: Part A. 2007;38:1710-1721.

Rambo, M.K.D.; Amorim, E.P.; Ferreira, M.M.C. Potential of visible-near infrared spectroscopy combined with chemometrics for analysis of some constituents of coffee and banana residues. Anal. Chim. Acta, 2013;775:4149.

Rambo, M.K.D.; Ferreira, M.M.C. Determination of Cellulose Crystallinity of Banana Residues Using Near Infrared Spectroscopy and Multivariate Analysis. J. Braz. Chem. Soc. 2015a;1-9.doi: http://dx.doi.org/10.5935/01035053.201501 18

Rambo, M.K.D.; Schmidt F.; Ferreira, M.M.C. Characterization of Brazilian biomass sources for biorefinery opportunities. Talanta. (2015b) Doi:

Brereton, R. G. Chemometrics: Applications of Mathematics and Statistics to Laboratory Systems, Ellis Horwood, Chichester, 1993.

Wold, S.; Esbensen, K.; Geladi, P. Principal Component Analysis. Chem. Intell. Lab. Syst. 1987; 2:37-52.

Guimarães, J. L.; Frollinni, E.; Silva, C.G.; Wypych, F.; Satyanarayana, K.G. Characterization of banana, sugarcane bagasse and sponge gourd fibers of Brazil. Ind. Crop Prod. 2009;30:407-415. 